\title{
Combining DNA and RNA analyses enhances non-invasive early detection of cutaneous melanoma
}

Stephanie R Jackson Cullison, MD, PhD ${ }^{1}$, Laura K Ferris, MD, PhD², Zuxu Yao, PhD ${ }^{3}$, Claudia lbarra ${ }^{3}$, Michael D Howell, PhD ${ }^{3}$, and Burkhard Jansen, MD 3

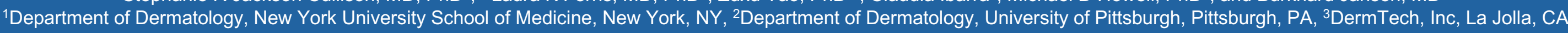

\section{SYNOPSIS}

The Pigmented Lesion Assay (PLA) is a gene expression test enhancing early melanoma detection. The test uses a proprietary non-invasive sample collection platform to objectively rule out melanoma and guide biopsy decisions. The PLA has been evaluated in over 60,000 patients with approximately $90 \%(54,000)$ of patients avoiding surgical biopsies due to negative results. The test's negative predictive value of $>99 \%$ has been validated in long-term follow-up studies. Combined with the rapid and painless application, the PLA is an attractive solution that misses fewer melanomas while reducing costs. Clinicians follow the guidance of the test in $98 \%$ of cases corroborating high clinical utility. To further improve the high performance of the PLA, RNA and DNA analyses were combined in a new test termed PLAplus. PLAplus combines gene expression analyses for LINC00518 and PRAME with TERT promoter mutation analyses thereby elevating the test's overall sensitivity from $91 \%$ to $97 \%$. The individual sensitivity numbers of these genomic targets on cases with consensus diagnoses of melanoma were 84\% (LINC00518), 83\% (PRAME), and 73\% (TERT). Additional studies in real-world use cohorts $(n=1,415)$ demonstrated the presence of TERT promoter mutations in up to $24 \%$ of PLA positive and $12 \%$ of PLA negative tests. TERT 146G $>$ A mutations were the most frequently observed mutational change $(48 \%)$. TERT $124 \mathrm{G}>\mathrm{A}(30 \%)$ and TERT138G>A $(12 \%)$ as well as TERT the early detection of melanoma by combining DNA and RNA analyses or

\section{OBJECTIVES}

To summarize available data and assess the real-world use of combining LINC00518 and PRAME gene expression analyses with TERT promoter mutation mutation analyses.

METHODS

All clinical studies were IRB approved. Gene expression analyses were performed by RT-PCR as previously described. Mutation analyses were performed by Sanger sequencing.

\section{RESULTS}

Efforts to further improve the high performance of the PLA led to a 作 termed PLAplus.

PLAplus combines gene expression analyses for LINC00518 and PRAME (two targets overexpressed in melanoma) with TERT promoter mutation analyses (Figure 1) which elevates the test's overall sensitivity from $91 \%$ to $97 \%$ (Figure 2). Individual sensitivity numbers of these genomic targets on cases with consensus diagnoses of melanoma were $84 \%$ (LINC00518), 83\% (PRAME), and 73\% (TERT). PLAplus conservatively focuses on maximizing sensitivity while maintaining a high specificity of $62 \%$. Adding TERT promotor mutation nalyses to LINCO0518 and PRAME further increases the test's negative predictive value from $99.3 \%$ to $99.6 \%$.

Studies in real-world use cohorts $(n=1,415)$ demonstrated the presence of TERT promoter mutations in up to $24 \%$ of PLA positive and $12 \%$ of PLA negative tests. While the biologic significance of TERT 146G $>$ A mutations were the most frequently obser sties, TERT 140A

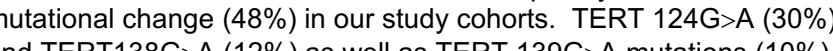
were also detected Increasing genomic atypia the morphogic atypia can be found on the spectrum of pigmented skin

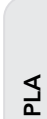
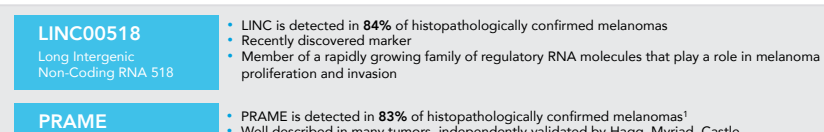
PRAME

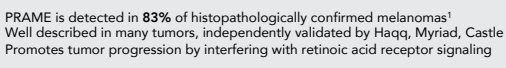
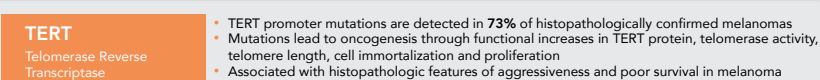

Figure 1: PLAplus genomic targets.

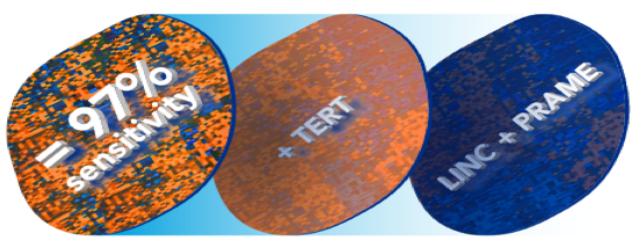

Figure 2: PLAplus combines gene expression and mutation analyses to further enhance early melanoma detection.

CONCLUSIONS

- Adding TERT promotor mutation analyses to LINC00518 and PRAME gene expression analyses further increases test's sensitivity from $91 \%$ to $97 \%$.

- The individual target's sensitivity numbers are $84 \%, 83 \%$ and $73 \%$ for LINC00518, PRAME and TERT, respectively.

- Adding TERT promotor mutation analyses to LINC00518 and PRAME gene expression analyses further increases test's negative predictive value from $99.3 \%$ to $99.6 \%$.

Both PLA and PLAplus lend themselves to remote sample collection under physician guidance in teledermatology environments.

\section{REFERENCES}

1.Gerami P et al. Development and validation of a non-invasive 2gene molecular assay for cutaneous melanoma. J Am Acad gene molecular assay for cutaneo
Dermatol, 2017;76(1)114-120.e2

2.Ferris $L$ et al. Noninvasive analysis of high-risk driver mutations and gene expression profiles in primary cutaneous melanoma. J Invest

3.Jackson $S$ et al. Risk stratification of severely dysplastic nevi by non-invasively obtained gene expression and mutation analyses. SKIN, 2020, 4(2)

4.Robinson $\mathrm{J}$ et al. Caring for melanoma survivors with self-detected concerning moles during COVID 19 restricted physician access: a cohort study. SKIN, 2020, 4(3) 